\section{Año 2019-2020. urtea} N. ${ }^{\circ} 31-32 . z k$.

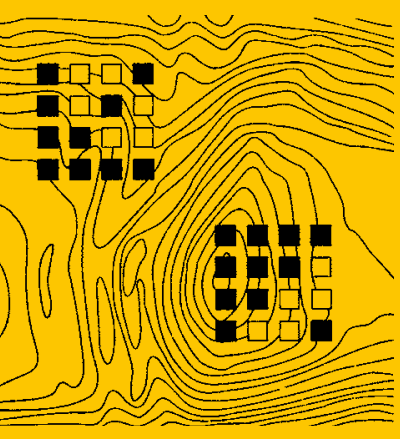

\section{TRABAJOS DE ARQUEOLOGÍA NAVARRA}

SEPARATA

\title{
Prospección: \\ batalla de Valdejunquera
}

Iñaki Sagredo Garde

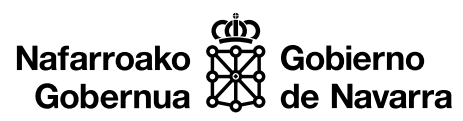

0000 


\section{Prospección: batalla de Valdejunquera}

Prospekzioa: Valdejunquerako gudua

Prospecting work: the battle of Valdejunquera

Iñaki Sagredo Garde

Gerente de Gestión Cultural Larrate S. L.

gc.larrate@yahoo.es

DOI: https://doi.org/10.35462/TAN31-32.17 


\section{RESUMEN}

En esta primera campaña de 2020, en el contexto del mil cien aniversario de la batalla de Muez, se ha realizado un estudio de los posibles lugares donde se pudo producir el combate entre las fuerzas omeyas de Abd al Rahman III y el ejército conjunto de Sancho Garcés I y Ordoño II. La prospección se ha realizado en varios espacios próximos a Riezu, Irujo y Muez, siendo hasta la fecha los resultados negativos dado el tipo de paraje en que nos encontramos: campos extensos de zonas de cultivo, con varios arroyos y desmontes. El proceso de estudio continuará en una segunda campaña, con una prospección en otros puntos del valle más próximos a la localidad de Muez.

Palabras clave: batalla; Abd al Rahman III; Muez; Córdoba; Valdejunquera.

\section{LABURPENA}

2020ko I. kanpaina honetan -Muezko guduaren mila eta ehungarren urteurrenaren testuinguruan- zenbait espazio aztertu dira jakiteko non izan ote zen Abd al Rahman III.aren soldadu omeiatarren eta Antso Gartzes I.ak eta Ordoño II.ak osatutako armadaren arteko borroka. Errezu, Iruxo eta Mueztik hurbil dauden espazio batzuetan egin da prospekzioa, eta orain arte emaitza negatiboak izan dira, lanean gabiltzan parajemota dela-eta: laborantzako landa zabalak, zenbait erreka eta erauzitako lur daudela. Azterketa honekin jarraitzeko, bigarren kanpaina bat eginen da, zeinetan prospekzioa eginen baita haraneko beste gune batzuetan, Muezko herritik hurbilago.

Gako hitzak: gudua; Abd al Rahman III.a; Muetz; Kordoba; Valdejunquera.

\section{ABSTRACT}

In the 2020 campaign, in the context of the anniversary of the battle of Muez, an study has been made in order to know the possible places where it could have been the combat between the omeya Abd al Rahman III's forces and the army of Sancho Garces I and Ordoño II. The prospecting has been done in some areas next to Riezu, Irujo and Muez, being negatives the results to date because of the type of location: crops and streams. The study will continue in a second campaing with more prospectings in some other areas of the valley near to Muez.

Keywords: Battle; Abd Rahman III; Muez; Cordoba; Valdejunquera. 
1. INTRODUCCIÓN. 2. ESTADO DE LA CUESTIÓN. 3. LA CAMPAÑA DE PROSPECCIÓN. 4. PROPUESTA DE ESTUDIO A FUTURO. 5. CONCLUSIONES. 6. BIBLIOGRAFÍA.

\section{INTRODUCCIÓN}

La batalla de Valdejunquera -26 de julio de 920 - es una referencia histórica para los estudios referidos al control Omeya de la península. La primera campaña de Abd al Rahman III en territorio cristiano supuso una derrota para los reyes Sancho Garcés I de Pamplona y Ordoño II de León. Sin embargo, aun siendo un desastre militar, no supuso un cambio significativo en la geopolítica del territorio. Ambos reyes continuaron con su expansión. Por otra parte, la victoria para el joven emir permitió afianzar su liderazgo y control en el Al Andalus, lo que años más tarde le permitiría llegar al califato.

El estudio del lugar del encuentro comienza con el itinerario seguido por las tropas musulmanas desde Córdoba hasta Di-Xarra, lugar próximo a Estella.

Por la crónica de Arib ibn Saad sabemos de la ruta seguida por el emir desde Córdoba es la siguiente: el 4 de julio partieron desde la capital del Al Andalús en dirección a Gormaz, Osma y Clunia. Luego movió la hueste hacia Tudela y, tras asegurar el territorio, se dirigió primero a Calahorra y luego hacia Cárcar. Cruzó el río Ebro el 23 de julio.

Desde Cárcar tomó el rumbo norte, siguiendo el cauce del río Ega, hasta el punto de Di-X-Xarra, zona próxima a Estella (Lizarra). Tras producirse un primer combate en este lugar, el 24 de julio Abd al Rahman III tomó la ruta hacia el norte, siguiendo a las tropas de Sancho, en dirección al valle de Guesálaz, donde se presentó la batalla no lejos de Muez. La victoria del emir fue aplastante según las crónicas. 


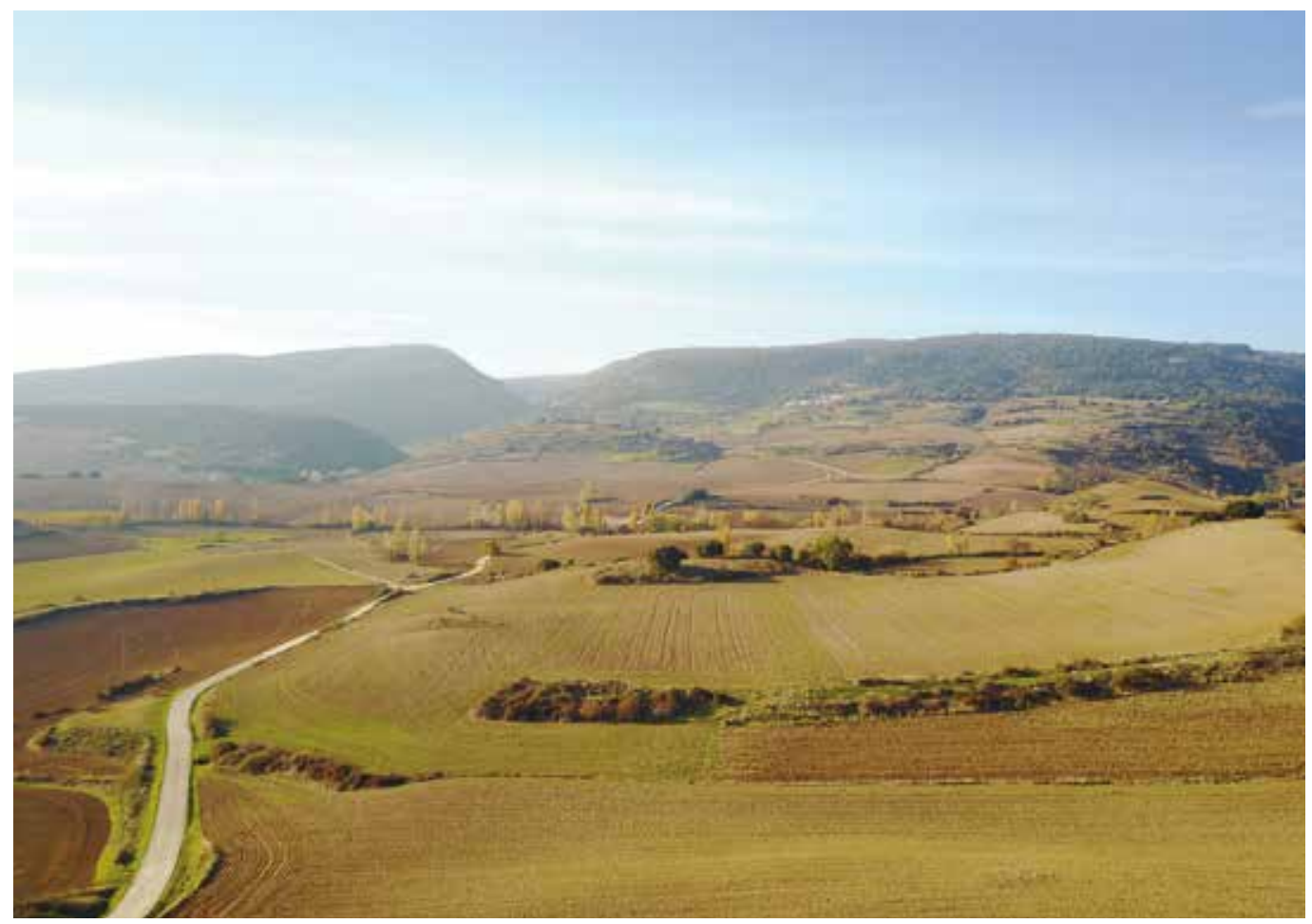

Figura 1. Toma áerea de la zona de estudio.

\section{ESTADO DE LA CUESTIÓN}

Prácticamente todos los autores que han profundizado el tema sitúan el lugar de la batalla en este punto, pero sin concretar mucho más.

Otros, sin embargo, se inclinan en el lugar de Mues, anotando la lógica de la batalla y la crónica de Sampiro donde anota el lugar de Mohis como punto del combate.

Para el estudio que presentamos se ha realizado previamente un análisis de puntos donde pudo producirse en encuentro. Estos son Junquera en término de Los Arcos; Junquera en Mendavia, no lejos del castillar, y en Mues, cerca del paso del Congosto, donde se estudió un castro en la parte superior que conserva las murallas y controla este paso estratégico, punto posible donde esperaron a las tropas de Abd al Raham.

Sería de gran interés analizar estos lugares con una metodología arqueológica para ampliar esta investigación.

Respecto a la localidad de Muez no hay indicios de castillo, aunque hay algunas referencias toponímicas y un dato como tenencia en el siglo XI. Por otra parte, se documenta un palacio desde 1417, el cual pudo tener cierta continuidad con las defensas más antiguas. 


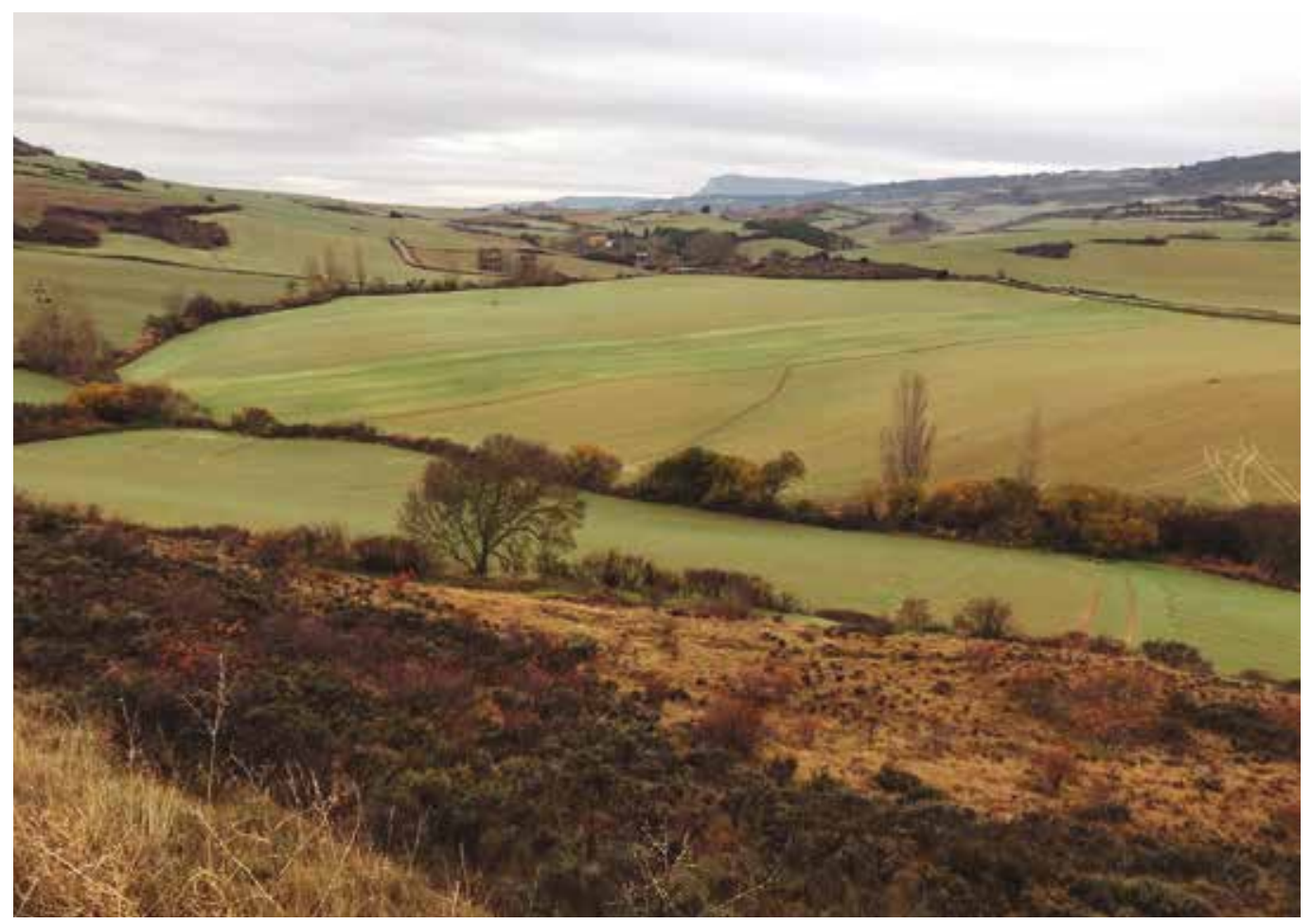

Figura 2. Campos próximos al río Ubagua.

Otra posibilidad de interés es el estudio del contexto del castillo de Oro, a escasos cinco kilómetros, como hipotético bastión refugio en esa batalla. No debemos olvidar que las tenencias eran distritos que abarcaban una amplia extensión, por lo que no se puede descartar el castillo de Oro como parte de la tenencia de Muez y que las fuentes lo mencionaran como tal siendo en realidad el situado en la Peña Grande o castillo de Oro.

El cronista del reino José de Moret (1615-1687), en su obra Anales del reino de Navarra, estudia el posible lugar de la batalla de Valdejunquera, situándolo en un campo próximo a Muez, exactamente entre Irujo al mediodía (sur) y la sierra de Andia al septentrión (norte). Es por tanto un lugar situado en un campo denominado Arreba, entre las localidades de Irujo y Riezu, enmarcado el entre la altura de Errezumendia y el regacho Obantzea.

Este espacio es una zona llana, extensa, con algo de pendiente hacia el río Ubagua (de norte a suroeste). Moret, con posiblemente más datos referidos al lugar anotaban lo siguiente:

Existen muchos rastros hoy en día de la batalla, levantándose con los arados bien frecuentemente lengüecillas arpadas de saetas, hierros de lanzas, pedazos de espuelas, trozos de frenos y algunos dorados todavía y con labor antigua y alguna vez con esmaltes de azul y oro (Moret, 1988, p. 186). 


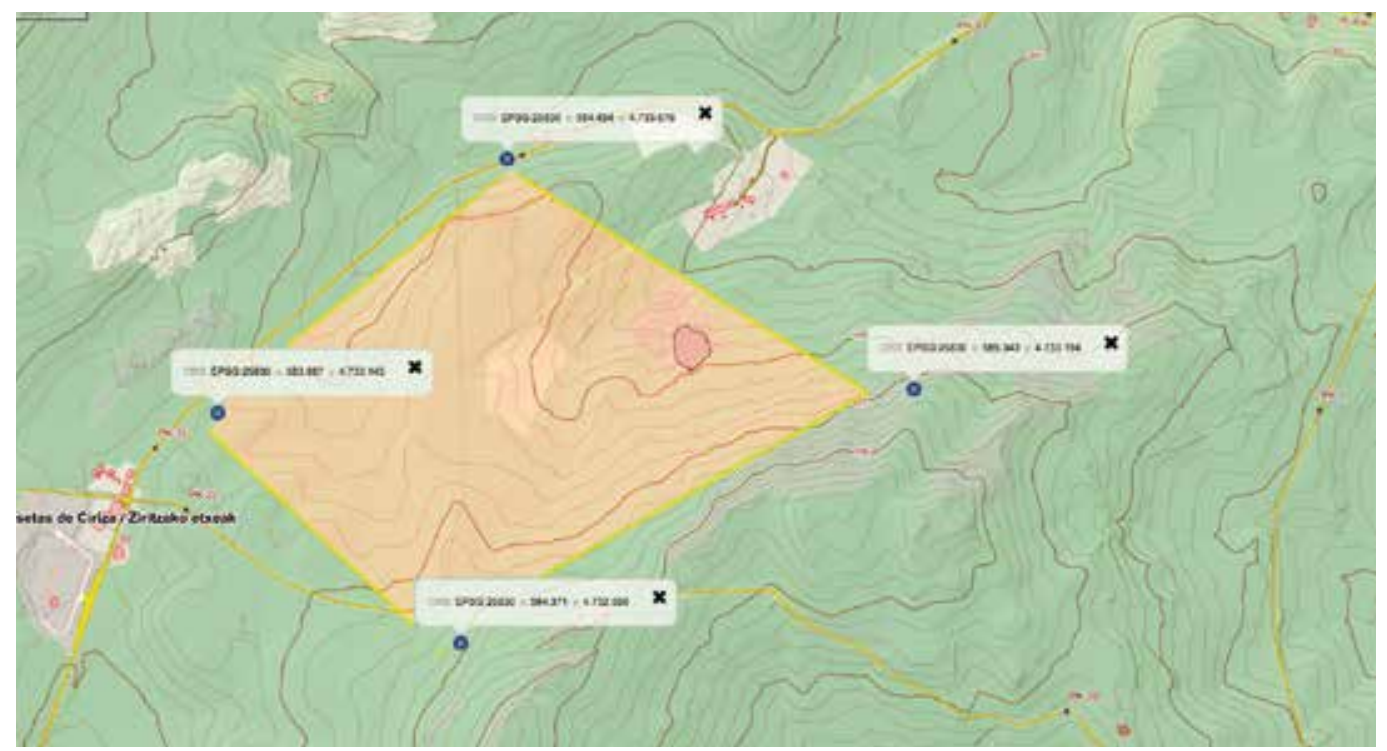

Figura 3. Primera zona de estudio en las proximidades de las denominadas casetas de Ciriza.

En el estudio que realizamos en este lugar, pudimos localizar restos de aperos de labranza pero ningún indicio medieval. No obstante, la zona es extensa y el lugar estudiado, como inicio de estudio, se situaba en un sector más próximo al río Ubagua. Es por tanto necesario seguir con la investigación en este sector en próximas campañas.

\section{LA CAMPAÑA DE PROSPECCIÓN}

Se realizó una prospección en una extensa área, próximo al lugar denominada «Las casas de Ciriza», donde próximo hay una referencia toponímica a mimbrales y a Moroa. El espacio es zona de labranza, delimitado por la carretera que se dirige a Riezu en su parte norte y al oeste con los montes de escasa altura que pertenecen a Villanueva de Yerri.

Para este trabajo se alquiló un Magnetómetro SBL 10 de Georeva que permite alcanzar grandes profundidades para la localización de objetos magnéticos, férricos. La prospección, como era de esperar, no aportó datos de interés. El 80 \% de los objetos fueron elementos de aperos de labranza, como hierros de enganche, puntas de arar, herraduras de caballerizas, pasadores, etc. No encontramos elementos medievales. Esta cuestión ralentizó el trabajo y nos centramos como segunda fase a la exploración del campo de Arreba, zona donde pudo desarrollarse el combate según la crónica de Moret. De igual forma el resultado fue negativo. No obstante, dicho campo tiene gran espacio y será precisó realizar más campañas para la posible localización del lugar de encuentro armado. 


\section{PROPUESTA DE ESTUDIO A FUTURO}

El proyecto debe continuar con más campañas. De esta primera fase hemos podido concretar el lugar más lógico para el encuentro entre los dos ejércitos, dado las alturas, la cañada que se dirige hacia la Sierra de Sarbil y la crónica de José de Moret.

Como se muestra en el siguiente mapa, el cuadrante de estudio se delimita por varios yacimientos que debemos estudiar: Errezumendi (Riezu-Yerri); Muez; Campos próximos a Muez y el castillo de Oro, en Salinas de Oro.

\section{CONCLUSIONES}

Entendimos desde el principio que el hallazgo de evidencias era muy difícil. A la dificultad añadida de intervenir en zonas muy removidas durante siglos por su uso como campos de labranza, hay que unir el punto exacto del encuentro. La extensión del posible lugar del encuentro es muy amplia.

Pero, el trabajar en varios puntos, ha permitido estudiar la zona, interpretando su geografía para localizar el punto idóneo donde se produjo el choque entre ambos ejércitos.

Para llegar a esta conclusión analizamos el recorrido que pudo utilizar la cañada que desde Estella, por Arandigoyen, Murillo de Yerri, Ugar, Casas de Ciriza, y tras cruzar el río Ubagua, se dirigía hacia Arguiñano-Irujo, con dirección a la sierra de Sarbil.

Un lugar interesante es el campo de Arreba, amplia extensión próxima a Riezu, que se llega tras cruzar el río Ubagua.

Respecto a la campaña arqueológica, los resultados son negativos. No se han encontrado evidencias de la batalla pero, el proyecto, dada la extensión del terreno, debe continuar con al menos una o dos campañas más.

Algunas zonas sugeridas son los campos próximos a Muez y Villanueva, además de la zona de Arreba.

\section{BIBLIOGRAFÍA}

Cañada, A. (1985). Revisión de la Campaña de Muez. Año 920. Principe de Viana, $174,127-143$.

Moret, J. (1988). Anales del Reino de Navarra, t. II (edición de S. Herreros Lopetegui). Pamplona: Gobierno de Navarra.

Lacarra, J. M. (1940). Expediciones musulmanas contra Sancho Garcés (905-925). Príncipe de Viana, 1, 41-70. 